\title{
Universidad y universalidad: aportes de la opción decolonial al estudio de políticas universitarias para la inclusión de estudiantes indígenas
}

University and universality: contributions of the decolonial option to
the study of university policies for the inclusion of indigenous students

\section{Por: Rodríguez, Nuria Macarena}

Instituto de Investigaciones en Ciencias Sociales y Humanidades (ICSOH-UNSa-CONICET)

Salta, Argentina

Email: nuria.macarena.rodríguez@gmail.com

https://orcid.org/0000-0003-3692-294X

Fecha de recepción: 15/03/2021

Fecha de aprobación: 20/05/2021

DOI: $\underline{\text { http://dx.doi.org/10.30972/nvt.1715354 }}$

\section{Resumen}

El presente artículo consiste en la discusión de categorías analíticas como inclusión, interculturalidad, universalidad, universalidad y colonialidad, todas en relación a las universidades argentinas y a las políticas institucionales y estatales orientadas a favorecer el acceso y la retención, en general, y de estudiantes indígenas, en particular. Para ello, se parte del trabajo realizado en el marco de la tesis doctoral de la autora y de problematizaciones teóricas que se desarrollaron en la misma. Este trabajo consiste, entonces, en un intento por articular los aportes de las teorías críticas al MCMC, específicamente la opción decolonial, con emergentes del trabajo de campo realizado para la tesis y de investigaciones que abordaron casos similares.

\footnotetext{
* Este artículo se constituye en parte de la tesis doctoral de su autora, titulada "Interculturalidad y Universidad: Desarrollo, Avances y Perspectivas del Proyecto de Tutorías con Estudiantes de Pueblos Originarios en la Universidad Nacional de Salta". Además, dicha tesis y, en consecuencia, este trabajo, se desarrolla en el marco del Proyecto de Investigación Institucional para Unidades Ejecutoras del CONICET "Territorialidad y poder: Conflictos, exclusión y resistencias en la construcción de la sociedad de Salta".
} 


\section{Nuria Macarena Rodríguez}

Palabras clave: Decolonialidad, Inclusión, Interculturalidad, Universidad, Universalidad.

\section{Abstract}

This article consists of the discussion of analytical categories such as inclusion, interculturality, universality, universality and coloniality, all in relation to Argentine universities and institutional and state policies aimed at favoring access and retention, in general, and of indigenous students. , in particular. To do this, it starts with the work carried out within the framework of the author's doctoral thesis and the theoretical problematizations that were developed in it. This work consists, then, in an attempt to articulate the contributions of the critical theories to the MCMC, specifically the decolonial option, with emerging from the field work carried out for the thesis and from research that addressed similar cases.

Keywords: University, Universality, Decoloniality, Inclusion, Interculturality

\section{Cómo citar este artículo:}

APA: Rodríguez, N. M. (2021). Universidad y universalidad: aportes de la opción decolonial al estudio de políticas universitarias para la inclusión de estudiantes indígenas. Nuevo Itinerario, $17(1), 192-225$.

\section{Introducción}

El Modelo Civilizatorio Moderno Colonial (en adelante, MCMC) legitima ciertos conocimientos y prácticas -los occidentales, impuestos como universales- en detrimento de otros -los que no responden a los parámetros eurocéntricos y por tanto son negados-. Este proceso se desarrolla a partir de la colonialidad del saber (Lander, 2000) y del poder (Quijano, 2000), expresada en la generalidad de las instituciones que conforman las sociedades occidentales.

Históricamente, la educación formal fue uno de los bastiones de este modelo y las universidades no son la excepción. El alcance de esta forma hegemónica de producción de subjetividad se expresa en la formación de profesionales en estas prestigiosas instituciones. Sin embargo, es la misma educación empleada para la 
Universidad y universalidad: aportes de la opción decolonial al estudio de políticas universitarias para la inclusión de estudiantes indígenas

(re)producción del Modelo la que puede ser una herramienta que permita producir cambios, para emancipar a los sujetos, y en tal sentido, construirse en una "educación como práctica de la libertad" (Freire, 1987, p. 74).

Los procesos de visibilización de luchas sociales, movimientos anticapitalistas, campesinos e indígenas, las disidencias sexuales, los feminismos, entre otros, se erigen como intersticios que avizoran una crisis de legitimidad del MCMC. Esta situación muestra la posibilidad de otras formas de conocer, de estar y de ser en el mundo, diferentes de las hegemónicas, y pone en cuestión los relatos modernos. Nos encontramos, entonces, ante procesos que resquebrajan los fuertes cimientos del MCMC en sus tres facetas: el capitalismo, la colonialidad ${ }^{1}$ y el patriarcado (González, 2020).

La naturaleza de este Modelo resulta invisibilizadora, expulsora y excluyente de todo aquello que no sea posible de asimilar a sus parámetros. Esto se expresa en las instituciones educativas, en general, y en las universidades, en particular, que, investidas de inclusivas y accesibles para todos y todas, en ocasiones terminan siendo excluyentes para muchos.

En el caso particular de los pueblos indígenas, esto se relaciona, además, con las tensiones entre las expectativas de inclusión y las de distinción (Novaro y Hecht, 2017), mediante las cuales estos grupos ponen en cuestión el MCMC, exigiendo ser incluidos en su diversidad, en su particularidad, incluidos con su bagaje cultural, lingüístico y conocimientos previos, y no a condición de abandonar sus culturas. Esta forma de concebir la inclusión, que no implica la superioridad de un grupo -inclusorsobre otro -a ser incluido-, sino un diálogo entre culturas, es la que hemos decidido llamar inclusión en términos interculturales, ya que se sustenta en la idea de diálogo. Esta idea es la característica central en el concepto de interculturalidad, comprendida como encuentro de autopoiesis de las culturas, de convivencia y comunicación entre

\footnotetext{
${ }^{1}$ Emplearemos indistintamente los términos "colonialidad" y "colonialismo" para referirnos al proceso de ocupación territorial por medios coercitivos (militares, políticos, económicos) y a la imposición del imaginario cultural europeo que perdura (Maldonado, 2007). Sin embargo, advertimos la distinción conceptual de entre ambos términos, pues el colonialismo refiere a "períodos históricos específicos y a lugares de dominio imperial", mientras que la colonialidad "denota la estructura lógica del dominio colonial" (Mignolo, 2005, p. 33).
} 
ellas (Tintaya Condorí, 2003). Así, la interculturalidad -en su concepción crítica requiere la transformación de las estructuras, instituciones y relaciones sociales, y la construcción de condiciones de estar, ser, pensar, conocer, aprender, sentir y vivir otras (Walsh, 2010).

La educación, aun siendo una consecuencia de la colonialidad y reproductora de la misma, puede generar espacios para permear, agrietar y tensionar el MCMC, cuestionando sus principios desde sus propias instituciones y reconfigurando los sentidos asociados a ellas. Las universidades no son la excepción, en ellas también se encuentran posibles intersticios que avizoran cambios. La investigación de estos intersticios, la producción teórica y el estudio específico de las experiencias que los generan, constituyen herramientas que posibilitan poner en cuestión el MCMC.

Precisamente, a tesis en la que se sustenta este trabajo consiste en estudio de una política educativa institucional en la Universidad Nacional de Salta (en adelante, UNSa), orientada a la atención y retención de estudiantes indígenas: el Proyecto de Tutorías con los Estudiantes de Pueblos Originarios (en adelante, ProTconPO). La investigación se enfoca en su estudio desde la perspectiva institucional, pero también y principalmente- analiza la experiencia de los propios sujetos en la universidad y su participación en el ProTconPO.

La UNSa es una Universidad Convencional, ubicada en la provincia con mayor diversidad de Pueblos Indígenas de Argentina. Sin embargo, no se reconoció institucionalmente en esta universidad la presencia de estudiantes indígenas sino hasta el año 2008. El proyecto que construimos como caso de estudio para nuestra investigación constituye la primera política de la universidad dirigida a estos grupos y la consideramos como una experiencia que puede posibilitar la apertura de nuevos horizontes institucionales en la UNSa. Al respecto, es importante mencionar que los estudiantes indígenas de esta universidad que participan en el ProTconPO, han conformado la Comunidad de Estudiantes Universitarios de Pueblos Indígenas (en adelante, CEUPO).

2. Inclusión en términos interculturales 
Universidad y universalidad: aportes de la opción decolonial al estudio de políticas universitarias para la inclusión de estudiantes indígenas

Existen múltiples definiciones de inclusión y el uso de esta categoría es profuso y muchas veces confuso. Para hablar de inclusión es necesario hacer referencia a la exclusión, porque la primera es consecuencia del reconocimiento de la existencia de la segunda y de la importancia de intervenir para atender a quienes resultan excluidos (Wigdorovitz de Camilloni, 2008). Entonces, ¿quiénes son los excluidos? Los pobres, los pobladores de zonas rurales remotas, las poblaciones indígenas, las minorías lingüísticas y religiosas, las personas con discapacidad, quienes padecen enfermedades contagiosas, todos aquellos que son diferenciados de los demás y -por tal razón- pesa sobre ellos un estigma social (Wigdorovitz de Camilloni, 2008). En pocas palabras, son aquellos que se alejan de los parámetros del MCMC y, por consiguiente, se invisibilizan, se niegan, se descartan.

Respecto al concepto de inclusión, C. Diez y otros (2015) nos recuerdan la definición de la Real Academia Española (RAE) en la cual se alude a poner algo dentro de otra cosa, meter, contener. Partiendo de esta definición y del análisis de los usos indiscriminados que se han hecho en distintos ámbitos (político, estatal, académico, etc.) del término, los y las autoras realizan críticas a los mismos en tanto no se pone en cuestión "el 'continente' (a dónde se incluye), el sentido (para qué) y el lugar reservado para los incluidos (clase) y en particular, la invisibilización de las modalidades por las que efectivamente están incluidos" (Diez et al., 2015, p. 38).

Los sentidos de la inclusión vinculados a las posibilidades de escolarización no se producen en el vacío, sino que forman parte de las diferentes visiones respecto de los motivos y los logros que se persiguen a través de la educación formal. Esto es más significativo si se piensa en la educación superior, que no es obligatoria. Además, implica reconocer que la inclusión y la exclusión son categorías analíticas que, en ciertas ocasiones, no llegan a dar cuenta de la complejidad de las trayectorias socioescolares reales de los sujetos, quienes se apropian de los espacios académicos de formas particulares, y en base a condiciones de existencia inequitativas, desiguales y diversas (Rodríguez y Ossola, 2019).

Las trayectorias escolares de los sujetos, entendidas como los recorridos de las personas en el sistema escolar, presentan características variadas en relación con la 
expectativa que supone el diseño de tal sistema (Terigi, 2008). Gran parte de los estudios existentes se basan en datos estadísticos que permiten delinear trayectorias escolares teóricas o ideales, que señalan los recorridos de los sujetos cuya trayectoria se ajusta a la prevista o normativizada (Terigi, 2008). Por otra parte, están las trayectorias escolares reales que no se ajustan necesariamente a las expectativas del sistema educativo formal, pues estudiantes desarrollan su escolarización de maneras heterogéneas, variables y muchas veces imprevistas (Terigi, 2008). Es importante señalar que entre los pueblos indígenas se evidencia un alejamiento pronunciado respecto de las trayectorias ajustadas a la normativa conforme los sujetos avanzan en edad y, más aún, cuando son correlacionados la edad y el año cursado o, específicamente, la edad y el año aprobado (Rodríguez y Ossola, 2019). De este modo, la repitencia, la sobre-edad y la deserción caracterizan las trayectorias escolares de los estudiantes indígenas en nuestro país. Así pues, si hablamos de inclusión educativa en relación a los pueblos indígenas, ésta adquiere características particulares.

Lo anterior es importante si se toma en consideración que, en los últimos años, en varios países, se pasó del discurso de homogeneización cultural a un reconocimiento de la diversidad étnica, otorgando una serie de derechos específicos a ciertos grupos.

La interculturalidad, en lo declamativo, forma parte -desde hace varias décadas- de la agenda de formulación y aplicación de políticas públicas.. En este punto, cabe señalar que al menos en Argentina, las políticas estatales no persiguen fines interculturales más que en su denominación, en tanto "se reconocen las particularidades étnicas, pero no se propician condiciones de modificación del tipo de relaciones sociales" (Diez, 2004, p. 196). Así pues, no propician diálogo, son focalizadas en determinados grupos, formuladas desde otros e impuestas. En términos de Walsh (2010), sostenemos que se trata de una concepción funcional; esta autora distingue tres perspectivas de interculturalidad:

- la denominada relacional, "que hace referencia de forma más básica y general al contacto e intercambio entre culturas, es decir, entre personas, prácticas, saberes, valores y tradiciones culturales distintas, los que 
Universidad y universalidad: aportes de la opción decolonial al estudio de políticas universitarias para la inclusión de estudiantes indígenas

podrían darse en condiciones de igualdad o desigualdad" (Walsh, 2010, p. 77). Así, podría interpretarse como sinónimo de otros conceptos que suelen asociarse como multiculturalismo o transculturalismo. Al respecto, Diez (2004) sostiene que "frente a la multiculturalidad, como categoría 'descriptiva', surge la interculturalidad, como categoría 'propositiva'” ( $p$. 194), aclarando, además, que no sólo se trata de esta distinción entre lo "descriptivo" y lo "propositivo", sino de la forma en que se describe desde la perspectiva multicultural, como si se tratara de colectivos separados.

- la que denomina funcional, "se enraíza en el reconocimiento de la diversidad y diferencia culturales, con metas a la inclusión de la misma al interior de la estructura social establecida" (Walsh, 2010, p. 77); al respecto, consideramos que una interculturalidad que es funcional al sistema dominante (eurocentrista, universalista, moderno, colonial, etc.), no es interculturalidad más que en su denominación.

- la denominada crítica, posición desde la cual se define a la interculturalidad como:

una herramienta, como un proceso y proyecto que se construye desde la gente -y como demanda de la subalternidad-, en contraste a la funcional, que se ejerce desde arriba. Apuntala y requiere la transformación de las estructuras, instituciones y relaciones sociales, y la construcción de condiciones de estar, ser, pensar, conocer, aprender, sentir y vivir distintas. (Walsh, 2010, p. 78)

Además, aclara que esta interculturalidad es algo por construir. En tal sentido, existen experiencias con algunas características propias de esta interculturalidad crítica.

La pretendida universalidad del MCMC resulta inconciliable con el concepto de interculturalidad, en tanto este último refiere no sólo a la co-existencia de múltiples culturas, sino a la posibilidad y necesidad de un diálogo, una interacción entre ellas, basado en la aceptación mutua, sin jerarquizaciones y fortaleciendo la diversidad. La multiculturalidad, la existencia -en este mundo en general y en América Latina en 
particular- de diversas culturas, es innegable ${ }^{2}$. Así, una inclusión que pretenda la adaptación -de los excluidos- al MCMC es excluyente y por ello, proponemos la noción de inclusión en términos interculturales.

Proponemos entonces pensar la inclusión desde una perspectiva decolonial, como opción al MCMC, que nos permita acercarnos a una inclusión intercultural. Quijano (1992), pionero de la opción decolonial, reclama la necesidad de reconocer la colonialidad del saber, para poder construir una nueva comunicación -y agregaríamos educación- intercultural.

Asimismo, Mignolo (2007) plantea que el giro decolonial significa la apertura hacia la libertad de pensamiento y hacia formas otras de vida. Negar la existencia de una racionalidad universal, no significa imposibilidad de diálogo. No hace falta "pensar igual" o tener las mismas lógicas de pensamientos o la misma racionalidad (universal) para poder comunicarnos, siempre y cuando seamos conscientes de esas diferencias y aceptemos que ninguna cultura es superior a la otra, de eso se trata la interculturalidad.

En este sentido, dada su naturaleza universalista, moderna y colonial, las universidades convencionales argentinas distan mucho de ser inclusivas en términos interculturales. Sin embargo, existen experiencias que permiten vislumbrar nuevos horizontes que, si bien pueden parecer utópicos, se constituyen en intersticios para poner en cuestión las facetas del $\mathrm{MCMC}^{3}$ e imaginar formas otras de inclusión en estas instituciones.

Al respecto, nos interesa señalar que no basta con formar indígenas para asegurar su protagonismo, si no se rompe con las diferentes formas de colonización (Luciano, 2009). De allí se desprende la importancia de aceptar que las iniciativas que

\footnotetext{
${ }^{2}$ Novaro (2006) realiza una distinción entre un nivel descriptivo de la interculturalidad, que es el que nosotros llamamos "multiculturalidad" y refiere a situaciones de hecho que se encuentran en espacios donde coexisten grupos distintos, y un nivel propositivo, que hace referencia a una situación que no existe, pero se considera deseable construir.

${ }^{3}$ Recordemos que las tres facetas del MCMC son el Capitalismo -en tanto sistema-mundo de sometimiento de los países pobres, en el que se reproducen las desigualdades socioeconómicas-, la Colonialidad -entendida como la estructura lógica de dominio que perpetúa la imposición del imaginario cultural europeo- y el Patriarcado -representado como un sistema de relaciones sociales instaurada por los varones, en el que se presupone una superioridad de éstos por sobre otros géneros-. Reconocemos entre estas tres facetas una relación intrínseca y entrelazada, que ha ido variando a lo largo del tiempo.
} 
Universidad y universalidad: aportes de la opción decolonial al estudio de políticas universitarias para la inclusión de estudiantes indígenas

buscan la inclusión en base a premisas de interculturalidad en el ámbito universitario deben considerar la desigualdad estructural de las sociedades en las que habitamos (Reygadas, 2007).

En ese sentido es que sostenemos la necesidad de poder pensar una inclusión que signifique un diálogo entre culturas o sectores sociales, en lugar de una inclusión acrítica que supone la superioridad de un grupo sobre otro. Además, es necesario contemplar la posibilidad de que el "excluido" puede no querer ser "incluido" en los términos que externamente se consideran los adecuados, en tanto esos términos implican colonialidad. Por ello, la idea de diálogo permite pensar en una inclusión que no sea en un solo sentido: desde el que incluye hacia el que "debe ser" incluido; sino en varios sentidos que impliquen incluir e incluirnos.

2. Universidad, Colonialidad y Universalidad

El concepto de inclusión que proponemos resulta, entonces, incompatible con la noción de universalidad. Consideramos importante problematizar esa noción en sus sentidos filosóficos, políticos y culturales, para comprender los regímenes del MCMC vigente. Además, realizamos un contraste de esta noción con la lectura que puede realizarse de ella en el ámbito las universidades nacionales argentinas como espacios en cuya discusión académica y epistemológica prima el universalismo moderno eurocéntrico.

El problema de la universalidad estuvo siempre presente en el pensamiento occidental, más aún después del advenimiento del cristianismo. Como bien señala Dussel (2000), recién con el descubrimiento de América -descubrimiento del Otro- esa universalidad abstracta que sostenía Europa desde la Antigüedad, se traduce en una mundialidad concreta. Esto significa que aparece en el horizonte una alteridad a la que es posible convencer, por las buenas o por las malas, de que hay una cultura "civilizada" que los supera y que es necesariamente universal, porque posee una razón y una verdad universalmente válidas.

Además, la conquista de Latinoamérica permite concretar la supremacía de Europa: "Su superioridad será, en buena parte, fruto de la acumulación de riqueza, 
experiencia, conocimientos, etc., que acopiará desde la conquista de Latinoamérica" (Dussel, 2000, p. 29).

Por otro lado, el convencimiento europeo del carácter civilizatorio de la modernidad llegó al extremo de culpabilizar a esa alteridad por resistir u obstruir el proceso que Europa sentía que estaba llamada a concretar: extender su civilización universalmente como la única verdad posible. Así se justifica la violencia, las víctimas son culpables y la estrategia civilizatoria moderna es inocente. De este modo, desde el MCMC, se construye a los sujetos no-europeos como objetos de los que se habla y separa radicalmente el mundo metropolitano y el mundo colonizado.

La noción de sujeto mencionada anteriormente también sirve de fundamento para sostener la idea de la superioridad de lo europeo:

Desde el punto de vista de este sujeto suprahistórico, podían clasificarse las distintas culturas y pueblos del mundo. Este sujeto se colocaba a sí mismo en oposición a aquellos que, como los bárbaros de Rousseau, se hallaban atrapados en sus tradiciones y eran incapaces de liberarse de sus instintos corporales (Jonsson, 2010, p. 112).

Entre estos últimos encontramos a las mujeres, las clases trabajadoras y los no europeos. Así pues, ubicar al Otro en el lugar del "bárbaro" y/o "incivilizado", coloca lo europeo como cultura redentora, permitiendo justificar y enaltecer su accionar, en tanto su fin es quitarlo de esa posición inferior, aunque para ello sea necesario emplear la violencia (epistémica, simbólica o física).

Entonces, los "valores universales" o los que vienen considerándose como tales, promovidos por el MCMC -y actualmente sometidos a crítica por muchas grupos disidentes, fueron suministrados por la cultura europea bajo la forma de derechos humanos que debían ser de aceptación general. Paradójicamente, para lograr la universalidad de esos valores -asociados con la cultura europea-, se violaron los derechos humanos, justificándose en lo ya analizado en líneas anteriores ${ }^{4}$.

\footnotetext{
${ }^{4}$ Para ejemplificar la contradicción intrínseca de esta imposición de valores, sin entrar en el terreno económico, basta citar la revolución de los esclavos negros en Haití, que fue el primer movimiento independentista de la América Latina a fines del siglo XVIII y comienzos del XIX, y que se inspiró en los ideales de la Revolución Francesa y su Declaración de los Derechos del Hombre. La Francia revolucionaria y jacobina mandó a sofocar esta revolución en abierta contradicción con aquellos supuestos ideales de libertad, igualdad y fraternidad. Esto
} 
Universidad y universalidad: aportes de la opción decolonial al estudio de políticas universitarias para la inclusión de estudiantes indígenas

La conciencia de una razón universal, que le es propia al pensamiento europeo, se pone de manifiesto con toda su virulencia en la conquista y colonización de América Latina. Si bien los ideales de la ilustración habían descubierto la dignidad del Otro en el plano ético, tales ideales no se aplicaron en la colonización de América Latina y, muy por el contrario, sirvieron para justificar la negación del Otro en su humanidad. Esto tuvo, al menos, dos consecuencias que se manifiestan hasta el presente: en primer lugar, la exclusión y -en muchos casos- la eliminación de pueblos indígenas; en segundo, la generación de un estrato social criollo -que ocupa las posiciones de poderque reivindica, sostiene y aplica aún los paradójicos y contradictorios ideales universalistas constitutivos y reproductores del MCMC (Rodríguez, 2018).

El Estado, como actor moderno, sigue imponiendo el MCMC en sus tres facetas. Así pues, nos interesa recuperar el aporte que realiza el pensamiento decolonial para pensar acontecimientos en contextos donde todavía perviven estructuras de dominación colonial (Quijano, 2000).

Como mencionamos, el ámbito privilegiado para la transmisión de estos valores, supuestamente universales, es la educación formal. Desde su surgimiento, la Escuela ha sido uno de los principales bastiones para adoctrinar a los sujetos hacia los fines ponderados por las clases dirigentes. Así, por ejemplo, en las instituciones educativas se transmite como "historia universal" la historia de la civilización mediterránea y europea, mientras se ignora a los pueblos originarios como sujetos históricos, incluyendo -en el mejor de los casos- algunas cuestiones referidas a las culturas Azteca, Maya e Inca, también desde una perspectiva europea:

La imposición de un pensamiento único ejercida por la colonialidad epistémica impregna todos los ámbitos de la vida cotidiana y, sobre todo, se concreta y sistematiza en el terreno de la educación en todos sus niveles. Desde el jardín maternal hasta la posgraduación, el sistema se retroalimenta a sí mismo, fortaleciendo en distintas dimensiones y con diversas estrategias el conocimiento único, una idea de nación y de ciudadanía que excluye a amplios sectores de la

demuestra una vez más que la cuestión del lenguaje genera el problema: términos como universalidad, razón, verdad, justicia y justificación provienen de hechos que se hacen pasar por conceptos, tanto como los principios de la Revolución Francesa. 
sociedad y que sigue -como en tiempos coloniales- controlando con la cruz, el lenguaje y el valor económico (Palermo, 2014, p. 135).

Particularmente, las universidades contribuyen a este colonialismo, en tanto se constituyen como espacios en los que prima el universalismo moderno eurocéntrico. Pero, recordemos también que son estos mismos espacios desde donde se puede cuestionar al MCMC y construir prácticas liberadoras.

\section{Universidades Argentinas}

Las primeras universidades surgieron a principios del siglo XIII en Europa, como sostiene Buchbinder (2006): “aunque las universidades fueron creadas entonces, tenían raíces antiguas y reconocían antecedentes en las escuelas formadas en las catedrales para instruir al Clero" (p. 1). Así pues, las características, formas de organización y estructuras de las universidades conservan rasgos muy antiguos y es por ello que el conocimiento de sus orígenes y su evolución nos permite explicar muchos de sus rasgos contemporáneos (Buchbinder, 2006).

Asimismo, Buchbinder (2006) comenta que, hacia principios del siglo XIX, la mayoría de las universidades occidentales seguían conservando los rasgos adquiridos durante el medioevo. Recién durante el mencionado siglo, las universidades europeas comienzan a evolucionar, adoptando dos grandes modelos: por un lado, el que concebía a la universidad como el ámbito para el desarrollo y práctica de la ciencia; éste fue el modelo que se desarrolló en los principados alemanes; por otro, el que concebía a la universidad como ámbito para el desarrollo y la formación de los profesionales, construido en Francia en tiempos de Napoléon (Buchbinder, 2006). Así pues, en el siglo XIX se terminan de configurar las características de la universidad que perduran hasta nuestros días (Cupo, 2010).

En tanto las universidades argentinas son consecuencia de la colonización, su historia debe "comprenderse en el mismo contexto de evolución de las universidades occidentales" (Buchbinder, 2006, p. 2). Quizá la universidad sea una de las más fuertes instituciones coloniales del saber occidental.

Tal como plantean Coronado y Gómez Boulín (2015), las universidades argentinas "son organizaciones intrínsecamente conservadoras que responden a 
Universidad y universalidad: aportes de la opción decolonial al estudio de políticas universitarias para la inclusión de estudiantes indígenas

premisas del mundo de los siglos XIX y primera mitad del XX" (p. 31). En este sentido, están conformadas de acuerdo con un modelo humanista occidental. Entonces, la barrera que deben franquear los estudiantes universitarios, en general, y los estudiantes universitarios indígenas, en particular, es la de un saber que se postula como universal.

En la actualidad, el sistema argentino de educación universitaria se compone por 111 instituciones, de las cuales 61 son de gestión pública, 48 de gestión privada, 1 de gestión extranjera (Universitá Degli Studi di Bologna) y 1 de gestión internacional (Facultad Latinoamericana de Ciencias Sociales) (SPU, 2017).

Además, siguiendo los datos brindados por la Secretaría de Políticas Universitarias (en adelante, SPU) (2017), de las 61 universidades de gestión estatal, 57 son nacionales y 4 provinciales. Además, la SPU clasifica a estas universidades, según la cantidad de estudiantes, en grandes (más de 50.000 estudiantes), medianas (entre 10.001 y 50.000 estudiantes) y pequeñas (menos de 10.000 estudiantes). Las instituciones estatales son mayoritariamente medianas $(52,5 \%)$, seguidas por las pequeñas (36\%) y las grandes (11,5\%); la UNSa es una universidad mediana con 31.414 estudiantes (SPU, 2017).

Las principales políticas orientadas a garantizar el derecho a la Educación Superior han sido el acceso irrestricto -sin mediar exámenes selectivos y $\sin$ establecimiento de cupos $^{5}$ - y la gratuidad en las instituciones de gestión pública (García de Fanelli, 2014). Al respecto, resulta central destacar que estas instituciones suponen una igualdad de oportunidades que no favorece a quienes pelean su ingreso y permanencia desde posiciones iniciales de desventaja (Coronado y Gómez Boulin, 2015). Así pues, en tanto los jóvenes que ingresan a la universidad presentan condiciones -sociales, económicas, culturales, de género, étnicas, simbólicas, etc.heterogéneas, este principio de igualdad "no sólo es insuficiente, sino que puede promover aún más la desigualdad" (García de Fanelli, 2014, p. 4).

Los datos estadísticos demuestran que Argentina presenta una alta tasa de matriculación en el nivel superior -probablemente producto de las políticas de acceso

\footnotetext{
${ }^{5}$ Algunas universidades incorporaron exámenes o cursos de ingreso selectivos, no es el caso de la UNSa.
} 
irrestricto y gratuidad a las que aludimos-: "en el año 2012, la Tasa Bruta de Escolarización Superior (TBES) entre los jóvenes de 20 a 24 años era 75,4 \% en la Argentina" (Ministerio de Educación, 2014, citado en García de Fanelli, 2015, p.18). Sumado a esto, la Ley de Educación Nacional $N^{\circ}$ 26.206/2006 extiende la obligatoriedad de la educación hasta el nivel secundario y asegura que su cumplimiento se viabilizará mediante instituciones que se ajusten a los requerimientos locales y comunitarios, lo cual implicó la incorporación de jóvenes de sectores históricamente excluidos a la educación secundaria, de carácter históricamente selectivo.

Como consecuencia, años después, se evidencia un aumento significativo en la matrícula de las Universidades Nacionales, según los datos publicados por SPU: en el año 2006 se preinscribieron 358.763 ingresantes, en el 2012 -cinco años después de implementada la ley- el número asciende a 423.920 preinscriptos, la curva continúa en ascenso y en 2017 -último dato publicado por la SPU- se registran 516.305 preinscriptos (Fig. 2, donde se observa la curva completa de datos disponibles).

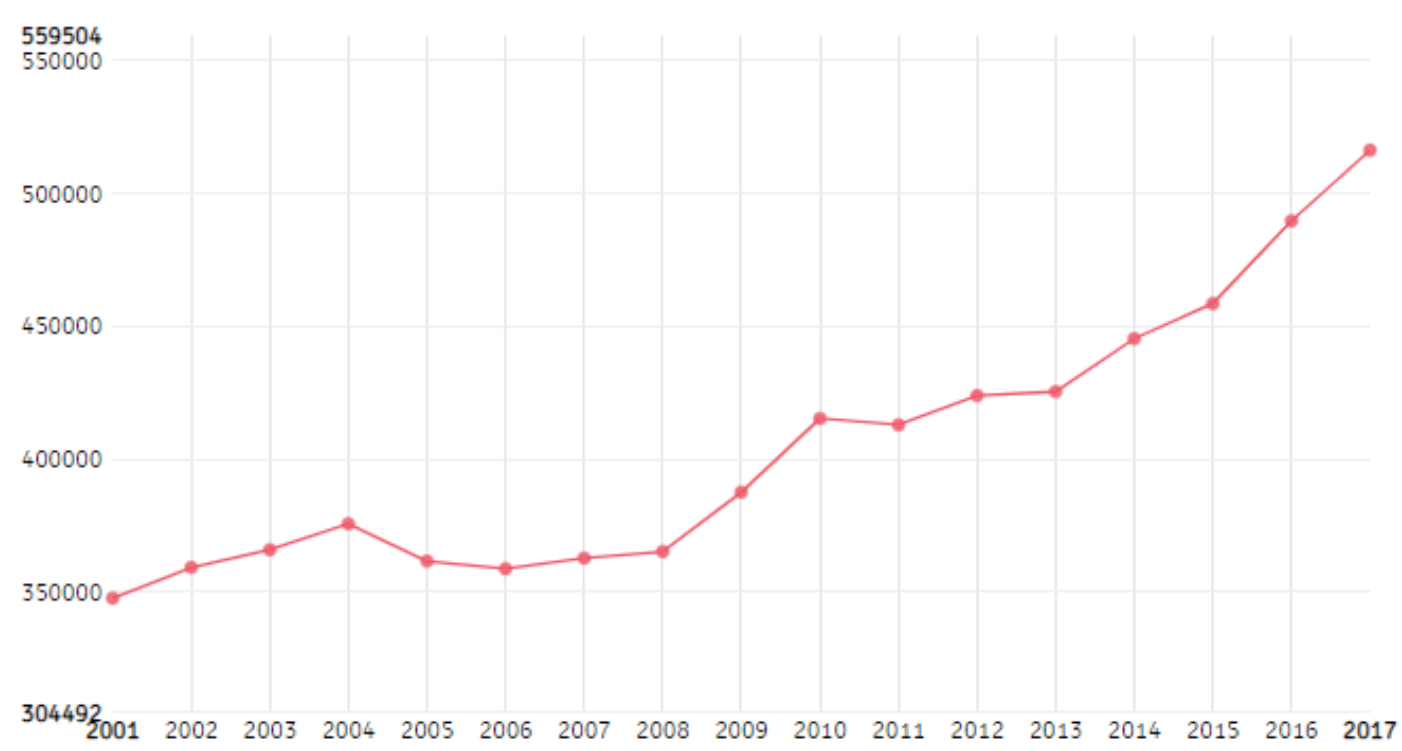

Figura 2. Evolución de la población estudiantil en carreras de grado y pregrado. Fuente: Secretaría de Políticas Universitarias (2017) 
Universidad y universalidad: aportes de la opción decolonial al estudio de políticas universitarias para la inclusión de estudiantes indígenas

Esta situación ha impactado en las expectativas sociales y educativas de los y las jóvenes de sectores desfavorecidos, así como en sus trayectorias de vida, permitiendo a muchos de ellos constituirse en la primera generación familiar en acceder al nivel secundario y, en algunos casos, a la universidad (Rodríguez y Sulca, 2020).

De este modo, Argentina es el país latinoamericano que presenta el mayor valor -en tasa neta- de participación del sector con menor ingreso -per cápita familiaren la universidad (García de Fanelli, 2014). Sin embargo, esta tasa coexiste con la menor probabilidad de culminar los estudios por parte de estos estudiantes. Así pues, los datos revelan que, si bien Argentina alcanza una adecuada cobertura de población que accede al nivel universitario, los problemas se evidencian en torno a la retención, rendimiento académico y graduación. En este sentido, la equidad en el acceso no tiene un correlato en el egreso, resultando el abandono una problemática que afecta en mayor medida a los grupos con menor nivel de ingreso per cápita familiar y a aquellos cuyos padres tienen menor nivel educativo alcanzado (García de Fanelli, 2014).

El concepto de "inclusión excluyente" (Ezcurra, 2011) resulta muy ilustrativo y esclarecedor para explicar el proceso mediante el cual el crecimiento de la matrícula se produce en un marco de procesos de igualdad que esconden la idea de que, al brindar igualdad de oportunidades, el éxito o el fracaso en los estudios universitarios dependerá de los méritos de los propios estudiantes. Este es un proceso que afecta a esa población desfavorecida en la distribución del capital económico y cultural que, con la masificación, logra ingresar en el sistema educativo superior (Ezcurra, 2011). En consecuencia, brindar iguales oportunidades a quienes llegan en desiguales condiciones crea una ficción de equidad que se traduce en una reproducción de las desigualdades.

Ahora bien, esa condición de "desfavorecidos" se relaciona también con que el MCMC imposibilita la presencia de saberes otros, culturas otras, formas de organización económica otras. Los conceptos de "capital económico" y "capital cultural" conciernen a las facetas del modelo, principalmente con la capitalista y la colonial, reproduciendo la idea de una cultura que tiene valor -la europea- y un orden 
económico en el que el capital económico se relaciona directamente con la capacidad adquisitiva en el sistema capitalista. Lo que sostenemos con esto es que la inclusión excluyente se relaciona -además de con la desigualdad - con la anulación de otras culturas, de otros saberes, que se produce en y con el MCMC, representado en un universalismo eurocentrista ${ }^{6}$.

La perspectiva decolonial permite -entre otras posibilidades- dar cuenta críticamente de las significaciones que la modernidad produce en quienes la experimentan en condición de subalternos. En ese sentido, sostenemos que la inclusión excluyente, puede ser explicada desde el concepto de universalidad que problematizamos. De modo que, complementamos el concepto de inclusión excluyente de Ezcurra (2011), sosteniendo que es también un proceso mediante el cual se incluye a los individuos, mientras se excluye sus saberes, culturas y modos de concebir el mundo, en tanto la universalidad es por naturaleza excluyente.

Es evidente que la masividad de las universidades tiene un correlato en la diversidad de los estudiantes que a ellas acceden, es decir que la gran cantidad de jóvenes que logran concluir el nivel medio e ingresar a la universidad presentan características heterogéneas. En consecuencia, y contemplando la insuficiencia del principio de igualdad de oportunidades, existe una tendencia a atender diferencialmente a quienes se encuentran en una situación inicial desigual, esto se hace a partir del diseño de políticas públicas e institucionales, entre las que se destacan aquéllas que promueven la incorporación de sistemas de tutorías en las universidades nacionales y los programas de becas para los estudiantes universitarios pertenecientes a hogares con bajo nivel de ingreso (García de Fanelli, 2015).

Para el análisis de la investigación realizada, propusimos exceder a la política prescripta y analizar las experiencias de los sujetos. Para tener una perspectiva más acabada, en relación al recorte y los objetivos de la investigación, se propone mover el foco desde lo institucional a lo subjetivo, es decir, pensar en los estudiantes como actores institucionales, en tanto usuarios de las universidades, pero también producto

\footnotetext{
${ }^{6}$ En este punto, un ejemplo es que los procesos de seguimiento y evaluación de los estudiantes se encuentran mediados por la escritura, ¿qué pasa entonces con las culturas ágrafas o aquellas donde prima una comunicación y aprendizaje a través de las imágenes?
} 
Universidad y universalidad: aportes de la opción decolonial al estudio de políticas universitarias para la inclusión de estudiantes indígenas

de éstas (Carli, 2012). Este movimiento nos habilita a pensar a las instituciones universitarias como espacios formativos que mantienen interlocuciones con el Estado y con las demandas de la sociedad y que produce graduados en determinadas profesiones y disciplinas, pero también como reservorio de experiencias que conforman la "vida universitaria" (Carli, 2012).

Los estudios disponibles acerca de las experiencias estudiantiles se centran en diferentes universidades de las consideradas "grandes" (SPU, 2017), en tanto reciben a la mayor cantidad de estudiantes, quienes incluso viajan de otras provincias para estudiar en ellas, lo que se traduce en características que no necesariamente pueden ser retomadas para analizar otras universidades. Así, notamos una vacancia de investigaciones acerca de estas experiencias en universidades con menos cantidad de estudiantes y menor tiempo de existencia. Entonces, nos interesa evidenciar la importancia de las investigaciones situadas, en especial cuando se trata de sistemas universitarios como el argentino, que presenta una gran heterogeneidad a su interior.

Como sostiene Carli (2012):

explorar las experiencias institucionales en un tiempo-espacio determinado hace posible una mirada a la vez interesada y crítica sobre esa construcción sedimentada que es la universidad pública, tensionada entre elementos del pasado, del presente y del futuro, entre elementos estructurales y situaciones epocales, entre elementos permanentes y posibilidades de cambio. (p. 25)

Si bien las universidades presentan rasgos característicos comunes, como los que desarrollamos en el apartado anterior, tienen también sus propias características: "no existe 'la' universidad, sino universidades con rasgos particulares, que invalidan a esta altura una lectura homogeneizante y simplificadora" (Carli, 2014, p. 16). Por ello, en esta tesis buscamos contribuir al conocimiento situado acerca de las experiencias de estudiantes indígenas en la UNSa, poniendo en suspenso las explicaciones universalistas tan características de los ámbitos universitarios.

5. Las Universidades argentinas y la inclusión de estudiantes indígenas

Resulta pertinente interrogarnos si las políticas estatales e institucionales orientadas a atender a los sectores que ingresan a las universidades en situación de 
"desventaja" -a las que referimos en el apartado anterior- atienden a la diversidad cultural. El desarrollo acerca de la naturaleza colonial de las universidades argentinas deja claro que no fueron pensadas para esta diversidad; sin embargo, existen experiencias que permiten avizorar nuevos horizontes.

Entonces, nos interesan en esta investigación las experiencias de un grupo en particular: los estudiantes pertenecientes a pueblos indígenas. Si bien no existe ninguna definición de pueblos indígenas que logre el consenso y la aceptación universal (Moro González, 2007), consideramos fundamental precisar a quiénes nos referimos cuando empleamos este término ${ }^{7}$.

Stavenhagen (2007) señala que "no existe una definición internacionalmente convenida de pueblos indígenas" (p. 38). Además, "definir para algunos pueblos indígenas, significa seguir siendo etiquetados, marginados y discriminados, por lo que tratan de evitar todo tipo de conceptualizaciones" (Civallero 2007, p. 8). Así, elaborar una definición de pueblos indígenas unívoca y acabada resulta una tarea difícil, pero además sería desacertado e ilegítimo intentar representar a la diversidad de pueblos indígenas en tal conceptualización. Tal pretensión representaría un acto de colonialismo, en tanto conllevaría a un borramiento de la diversidad cultural, encorsetando a los distintos pueblos en una única definición.

En la actualidad, "los pueblos indígenas reivindican la preponderancia de la aplicación del criterio de la autoidentificación" (Cloud, González y Lacroix, 2019, p. 61). Por ello, y en tanto compartimos la idea de que "ni al Estado ni a los científicos les corresponde definir quién es o no es indígena" (Trinchero, 2010), cuando nos referimos a pueblos indígenas $u$ originarios, aludimos a quienes se autoidentifican como tales.

Ahora bien, a los objetivos de la investigación, nos interesaron las experiencias de estos pueblos en las universidades. En las últimas décadas se han creado, en varios países latinoamericanos, universidades y otras Instituciones de Educación Superior (en adelante, IES) que, según los casos, se denominan indígenas y/o interculturales.

\footnotetext{
${ }^{7}$ También cuando empleamos el término pueblos originarios que es como se los nombra en el proyecto que analizamos en la Tesis de la que emerge este trabajo.
} 
Universidad y universalidad: aportes de la opción decolonial al estudio de políticas universitarias para la inclusión de estudiantes indígenas

Siguiendo a Mato (2015), tomamos la clasificación de las IES en tres categorías, teniendo en cuenta el tipo de relación de las mismas con los pueblos indígenas:

- Las universidades propias o universidades indígenas: han sido creadas por organizaciones o intelectuales indígenas y están dirigidas a estudiantes indígenas ya que su finalidad es la formación de intelectuales indígenas.

- Las universidades o IES interculturales: no han sido creadas por organizaciones indígenas, pero sí en colaboración con ellos e incorporando sus propuestas educativas. Si bien se orientan principalmente a estudiantes de pueblos indígenas, son abiertas a recibir a otros estudiantes, ya que la finalidad es generar egresados comprometidos con el respeto por la diversidad.

- Las universidades o IES convencionales: no han sido diseñadas para responder a demandas o propuestas de pueblos indígenas $y$ afrodescendientes.

Por lo que refiere a estas últimas, han surgido diversos tipos de programas orientados a la inclusión de personas indígenas en universidades "convencionales". En Argentina, estos procesos son relativamente más recientes que en varios otros países de la región (Mato, 2015).

Es así que los vínculos entre los pueblos indígenas y las universidades pueden ser variados, y en esta investigación interesan principalmente los que se dan a partir de programas destinados a la inclusión de estudiantes de pueblos originarios en universidades "convencionales", teniendo en cuenta que la apropiación e incorporación de esquemas pedagógicos provenientes del multiculturalismo clásico desafía profundamente la tradición universalista y "occidentalista" (Mignolo, 2000) de este tipo de instituciones académicas.

Mato (2015) señala el carácter monocultural de base de las IES al momento de formar profesionales provenientes de comunidades originarias. Al respecto, expresa que

es necesario evaluar cuál ha sido el papel de las Instituciones de Educación Superior "convencionales" en la formación de cuadros políticos, profesionales y técnicos provenientes de grupos sociales que sufren algún tipo de discriminación y obstáculos 
al ejercicio pleno de sus derechos, para que estén en mejores condiciones de construir más y mejor democracia. (Mato, 2015, p. 14 y15)

Asimismo, es importante el análisis acerca de la manera en que las oportunidades de formación que la universidad ofrece a la ciudadanía en general (sin distinguir grupos sociales) resultan pertinentes con la diversidad cultural. Es decir, si forman profesionales capacitados respecto de la diversidad cultural de sus respectivos países, que implica diversidad de visiones de mundo, valores, modos de producción de conocimiento, tecnologías, lenguas, etc., significativos para sus respectivos campos de acción (Mato, 2015).

Como hemos planteado, las mayores posibilidades de acceso a las universidades no necesariamente se traducen en una atención a la pluralidad. Resulta pertinente, entonces, desarrollar acciones orientadas a la permanencia y graduación de los estudiantes indígenas. Esta última no depende sólo de intenciones y voluntades de los sujetos, sino que también se encuentra condicionada por otros factores externos a los mismos (Hanne y Mainardi, 2013).

Entre las problemáticas que pueden influir en la permanencia y egreso de los estudiantes indígenas podemos mencionar, siguiendo a Hanne (2018) los siguientes:

- Aspectos vinculares: se relacionan con la diversidad en las formas de vincularse interpersonalmente. Así, los estudiantes indígenas se encuentran el a Universidad con la necesidad de establecer vínculos diferentes a los que acostumbran en sus lugares de origen. Además, los vínculos con sus familiares también se modifican por la distancia.

- El desarraigo: referente a las dificultades como consecuencia de dejar su comunidad de origen y trasladarse a otro espacio, está muy relacionado con los aspectos vinculares, pero implica también otros aspectos como los tiempos diferentes, la alimentación diferente, en algunos casos, viviendas diferentes, etc.

- Dificultades académicas: referidas a cuestiones relacionadas específicamente con las asignaturas de las carreras que estudian, pero también algunas más generales, como la complejidad de los textos, o la diferencia de lo estudiado con sus propias cosmovisiones. En este punto se suma también un aspecto vocacional, en tanto el 
Universidad y universalidad: aportes de la opción decolonial al estudio de políticas universitarias para la inclusión de estudiantes indígenas

sentimiento de estar cursando la carrera equivocada suele ser un motivo de abandono, en el estudiantado de nivel superior, en general, con características particulares en aquellos que sienten un compromiso particular con sus comunidades.

- Diversidad lingüística: esta problemática podría considerarse como específica de los wichí, en tanto son el único pueblo de la provincia que mantiene su lengua como primera lengua, mientras que en los otros la primera lengua es el español. Sin embargo, existen regionalismos que no son aceptados en la academia, lo cual extiende esta dificultad a otros estudiantes indígenas.

De esta manera, a pesar de los avances en el acceso a la educación superior de sectores históricamente excluidos -al menos en lo que respecta a Argentina, como correlato de la obligatoriedad de la educación secundaria-, este acceso coexiste con significativas brechas socioeconómicas y culturales, que atraviesan las trayectorias educativas de los y las estudiantes indígenas. A esto se le suma el carácter netamente moderno, colonial y occidentalizado de las instituciones de estos niveles y la carencia de un abordaje real desde una perspectiva intercultural.

Si bien, como plantea Rosso (2019) a nivel estatal no se ha avanzado significativamente respecto a las demandas indígenas de educación superior, la autonomía de la que gozan las universidades nacionales argentinas posibilita la implementación de políticas institucionales destinadas a la atención de las diferentes problemáticas de los estudiantes universitarios de pueblos indígenas. Actualmente, son ocho las universidades públicas argentinas que cuentan con alguna política de esta índole, ya sea mediante programas de becas y cupos especiales, mediante programas de apoyo académico y psicosocial, o mediante programas que combinan ambos (Guaymás, 2016).

Hanne (2015) señala que existe un interés de algunas universidades por incluir y abordar en sus agendas el tema indígena, desarrollando un genuino compromiso para con los estudiantes originarios mediante el diseño e implementación de políticas y estrategias que propician espacios inclusivos, en donde sus derechos puedan ser materializados en acciones concretas. Esta preocupación se advierte en

la atención que ha merecido la creciente presencia de pueblos indígenas en los ámbitos académicos, y la intención, ya sea de la Universidad en su conjunto, de 
algunas Facultades o equipos de cátedras, por favorecer la inclusión y permanencia de estos estudiantes mediante diversos tipos de beneficios y apoyos (...), procurando reducir las brechas de equidad y las deserciones. (Hanne, 2015, p.4).

En relación a esto, Ossola (2013) remarca que estas iniciativas de base étnica Iniciativas de Base Étnica (en adelante, IBE) en universidades nacionales forman parte de un conjunto de medidas que en sus enunciados, aplicaciones y fines expresan tensiones, pujas y reacomodaciones sociales, políticas y pedagógicas más amplias (Ossola, 2013). La noción de IBE refiere específicamente a las políticas y programas que buscan atender las necesidades y demandas de los indígenas en las instituciones de educación superior de tipo convencional.

Estas medidas se caracterizan por ser tomadas ad hoc y expresan relaciones de empatía y vínculos sociales entre los agentes de rango medio (generalmente encargados de formular el proyecto) y sectores de jerarquía en la universidad. Si bien logran posicionarse en el marco legal de la universidad, su implementación queda en manos de quienes se encuentran comprometidos, grupos reducidos de personas afines y sensibilizadas en la temática (Ossola, 2013).

Con los diferentes proyectos aprobados en las universidades, se intenta atender a las diversas problemáticas mencionadas anteriormente (Aspectos vinculares, desarraigo, dificultades académicas y diversidad lingüística), con el objetivo de mejorar las trayectorias educativas de los estudiantes indígenas. Hanne (2018) identifica dos aspectos principales que resultan, desde la perspectiva de los propios estudiantes indígenas, facilitadores de su permanencia en la universidad: las características personales (referidas al propio esfuerzo) y la presencia de otros sujetos significativos. Con estos últimos, se refiere a personas cuyo papel fue importante, facilitando las permanencias de los estudiantes indígenas en la universidad. A este respecto, -y en relación con nuestra investigación- resulta importante mencionar que entre esos "otros significativos", algunos estudiantes indígenas de la UNSa mencionan a los integrantes del ProTconPO. Con porcentajes inferiores, aparecen como aspectos facilitadores de las permanencias las siguientes categorías: Afectos y Familia, dimensiones académicas y formativas, y, con el menor porcentaje, los aspectos económicos. 
Universidad y universalidad: aportes de la opción decolonial al estudio de políticas universitarias para la inclusión de estudiantes indígenas

\section{Tutorías Universitarias}

Ahora bien, resulta pertinente esclarecer a qué referimos cuando hablamos de tutorías. Existen variadas acepciones del término, así como modalidades diferentes de tutorías. El presente trabajo de investigación aborda, concretamente, un caso de tutoría universitaria, la que puede definirse como un acompañamiento, orientación y apoyo al alumnado en su proceso de personalización de los aprendizajes y desarrollo de competencias a nivel personal y profesional, a lo largo de su trayectoria, con el horizonte dinámico del proyecto de vida, un proyecto personal y profesional (Lovato Fraile e Ilvento, 2013). De este modo, podemos pensar las tutorías como un vínculo socio-pedagógico entre diferentes actores universitarios, tendientes a personalizar la experiencia académica de los estudiantes.

Cuando hablamos de acompañamiento, nos referimos a un espacio de encuentro que "tiene carácter permanente de ensayo y puesta en práctica, para pensar con y a otros, que opera intermediando entre las condiciones reales de cada organización y las condiciones requeridas" (Coronado y Gómez Boulin, 2015, p. 112). Con esto queremos señalar que no concebimos a las tutorías universitarias como cerradas ni acabadas, sino que se encuentran en constante construcción. Es decir, que se construyen y reconstruyen como espacios interactivos y en los mismos vínculos interpersonales.

El momento en que se originan las tutorías institucionalizadas difiere según el nivel educativo al que nos refiramos. En el contexto universitario argentino, las tutorías existen desde los inicios mismos de las universidades, pero la función y los objetivos de éstas fueron variando según el contexto histórico y sociocultural en el que se desarrollaran, así como fueron cambiando -según esos contextos- los modelos de universidad en el país.

En la actualidad, la función del tutor en las universidades está vinculada a ayudar y cumplir tareas que complementan la enseñanza de los docentes; en estas modalidades actuales, hay mayor flexibilidad en el ejercicio de los roles de tutor y tutelado, donde las posiciones no son inmutables (Capelari, 2016) y las relaciones jerárquicas tienden a difuminarse. 
Es importante, además, tener en cuenta dos aspectos centrales de las tutorías con las que trabajamos: por un lado, que se trata de tutorías entre pares o tutorías entre iguales, es decir, que tanto los tutores como los tutelados son estudiantes de la misma universidad, lo que posibilita rotación en los roles de tutores y tutelados, así como cierta simetría en las relaciones; por otro lado, que la participación en las mismas es voluntaria, no obligatoria: "como toda ayuda, se ofrece, pero no se impone: uno de los principales retos consiste en implicar al alumnado" (Coronado y Gómez Boulin, 2015, p. 118).

La figura de tutores pares es valiosa "ya que se considera fundamental su experiencia en el recorrido universitario para brindar un acompañamiento apropiado en cuestiones académicas, institucionales y motivacionales" (Soto, Luján y Rosso, 2019, p. 8). Del mismo modo, se prioriza que estos roles sean desempeñados por estudiantes indígenas ya que "el tutor indígena posee esta doble "fortaleza", la experiencia en lo académico, por un lado, y la identidad cultural, por el otro" (Soto, Luján y Rosso, 2019, p. 9).

En algunos de las IBE cobra importancia también la participación de los referentes indígenas, aunque no es el caso del ProTconPO. Desde otras, entre ellas el ProTconPO, se intenta alentar el desarrollo de proyectos de extensión, en función de las demandas de las comunidades y organizaciones de pueblos indígenas de la región, este tipo de proyectos suelen contar con la participación de estudiantes indígenas, lo cual se constituye en una de las estrategias de permanencia. Asimismo, se busca promover y consolidar equipos de investigación acerca de la temática indígena, fomentando investigaciones intra e interinstitucionales sobre pueblos indígenas en educación, lenguas, salud, derechos, entre otros (Rosso, Artieda y Luján. 2016).

Pese a la importancia atribuida a los diversos programas y proyectos destinados a la inclusión de estudiantes indígenas en las universidades, es necesario mencionar que Hanne (2018) revela cómo desde la perspectiva de los estudiantes indígenas, la atención a la diversidad cultural en las Universidades Convencionales no representa una política instituida o no resulta compatible lo prescripto con lo actuado. En este sentido, hace referencia a las modificaciones que los estudiantes indígenas sugieren 
Universidad y universalidad: aportes de la opción decolonial al estudio de políticas universitarias para la inclusión de estudiantes indígenas

para los servicios universitarios: casi la mitad de los estudiantes que la autora encuestó, sugieren cambios en materia de políticas inclusivas. Luego, se encuentran las siguientes categorías, con porcentajes menores: servicios de bibliotecas y afines, aspectos administrativos, servicios tutoriales y de orientación, cuestiones de infraestructura y de recursos materiales y, por último, propuestas de extensión y prácticas extracurriculares.

Más allá de las críticas y posibilidades de mejora, es innegable la relevancia de estos programas y proyectos para favorecer la inclusión, retención y promoción de los estudiantes. Sin embargo, compartimos la idea de que es necesario "pasar desde las experiencias particulares -sostenidas por universidades de manera aislada- a la demanda de políticas de alcance nacional que garanticen el ingreso, permanencia y egreso de indígenas" (Artieda, Rosso, Luján y Zamora, 2017). Es decir que, más allá de lo valiosas que puedan resultar estas experiencias, se convierte en una necesidad el hecho de que la política inclusiva de nivel superior deje de estar sujeta a la buena voluntad e intencionalidad política de actores de las diferentes universidades, para ser responsabilidad genuina del Estado Nacional, a fin de garantizar el derecho a la educación a estos jóvenes.

Más allá de esta necesidad, nos interesa reconocer las experiencias de IBE que se desarrollan en diferentes universidades nacionales, como espacios desde los cuales se pueden iniciar cambios y cuestionar la lógica universalista y colonial. El valor de estos espacios cobra mayor claridad si los pensamos en relación a la raigambre colonial que caracteriza a estas instituciones. Así pues, nos permiten advertir nuevas formas de inclusión, superadoras.

7. Experiencias de estudiantes indígenas en la universidad.

Como adelantamos, en nuestra investigación, con el fin de una perspectiva más acabada de la política universitaria de inclusión para estudiantes indígenas en universidades convencionales, complementamos el análisis con la experiencia de los sujetos, actores principales de estos proyectos: los estudiantes universitarios indígenas. Siguiendo a Carli (2012), proponemos pensar en los estudiantes indígenas 
como actores institucionales, en tanto usuarios de las universidades, pero también producto de éstas. La autora sostiene que, para permanecer en una institución de nivel superior, los sujetos deben desplegar ciertas estrategias, mediante las cuales adquieren su condición de estudiantes universitarios, permitiéndose, de esta manera, ser exitosos en su formación, sorteando obstáculos y dificultades hasta llegar a culminar o avanzar en el plan de estudios de su carrera.

Así pues, a partir y en relación con los proyectos diseñados institucionalmente para la retención de estudiantes indígenas en las universidades, se desarrollan sus experiencias en tanto sujetos, desplegando sus propias estrategias para permanecer en estas instituciones.

Las IBE participan en la creación de escenarios propicios para el despliegue de nuevas formas de subjetividad y en la consolidación de un modo particular de asumir una identidad indígena en los términos de la institución: una "identidad supeditada" (Ossola, 2013). El proceso de acceso a mayores niveles de educación formal ubica a los jóvenes indígenas en posiciones sociales de ambigua definición, tanto en la universidad como en la familia y la comunidad indígena de origen (Ossola, 2013). De tal modo, la identidad supeditada (como espacio identitario sugerido por la universidad), es debatida por las y los jóvenes indígenas universitarios ${ }^{8}$ quienes "crean sus propios proyectos vitales, en los cuales conviven elementos provenientes de diferentes tradiciones culturales que son apropiados, reelaborados y resignificados a partir de sus necesidades en tanto jóvenes, indígenas y estudiantes universitarios" (Ossola, 2013, p. 14).

En cuanto a los sentidos de la profesionalización de los jóvenes indígenas, el debate pivotea entre dos posiciones contrapuestas: una que sostiene que el ingreso, permanencia y egreso de estudiantes indígenas en universidades convencionales conduce a la pérdida de su identidad indígena, y otra que indica que, como resultado

\footnotetext{
${ }^{8}$ Ossola (2015a) acuña la categoría de "Joven Indígena Universitario" porque permite retomar tres variables que caracterizan las experiencias vitales y formativas de sus interlocutores en la investigación (y también de los nuestros, en esta tesis): la etnicidad, la escolaridad y la pertenencia a un determinado grupo etario.
} 
Universidad y universalidad: aportes de la opción decolonial al estudio de políticas universitarias para la inclusión de estudiantes indígenas

de su trayectoria formativa, los jóvenes indígenas profesionales contribuirán al empoderamiento de sus comunidades (Ossola, 2013).

Además, los estudios acerca de la profesionalización de estos jóvenes se basan, generalmente, en las visiones de futuro formuladas por los adultos, quedando relegados los fines y objetivos perseguidos por los propios profesionales indígenas (Ossola, 2013). Muchas veces estos debates giran en torno a la idea de "retorno" a las comunidades, al respeto, Czarny, Ossola y Paladino (2018) señalan que el "retorno" no siempre se da por regresar materialmente a sus comunidades, sino que puede expresarse en la vinculación con la comunidad que no se interrumpe por la distancia física y permite que muchos jóvenes se sientan comprometidos con sus comunidades de procedencia. Permanecer en la universidad adquiere significados diversos para los estudiantes indígenas, entre los que contribuir a su comunidad es uno de los principales (Artieda, Rosso, Luján y Zamora, 2017). Como sostiene Santana Colin (2018), para el caso de las universidades:

Estos tránsitos escolares pueden convertirse también en una trinchera de resistencia donde los grupos indígenas que se encuentran en posición de subordinación y que han vivido históricamente discriminados, utilizan los conocimientos adquiridos en la universidad para sus propios fines, tanto personales como comunitarios (p. 108).

No se puede dejar de mencionar como factor influyente al menos en la permanencia -y quizá en el egreso- de los estudiantes indígenas la apuesta éticopolítica de hacer visibles sus identidades indígenas en las universidades. Este aspecto cobra importancia central en tanto no es objetivo inicial de los proyectos, sino que surge como iniciativa de los propios estudiantes: "los estudiantes están proponiendo acciones y asumiendo posicionamientos en una búsqueda por hacerse visibles como actores activos ante los demás miembros de la institución" (Soto, Luján y Rosso, 2019, p.12).

En relación a esto, es relevante mencionar que, durante el proceso de permanencia, habitar la universidad adquiere sentidos que van más allá del éxito académico, como establecer relaciones interétnicas y crear redes útiles no sólo para ellos mismos, sino para los futuros estudiantes indígenas universitarios, aportando a su rol de "pioneros" universitarios (Soto, Luján y Rosso, 2019). Las estrategias son, en este 
sentido, colectivas o comunitarias, no responden al objetivo de permanecer cada uno, sino de ir forjando espacios que faciliten la trayectoria a todos los estudiantes universitarios indígenas: "el 'ser universitarios', para estos sujetos, adquiere una significación ligada a una idea de superación de sí mismos como comunidad" (Luján, Soto y Rosso, 2018).

\section{Conclusiones}

En este trabajo hemos problematizado la forma en que las universidades convencionales responden, desde sus orígenes, a principios universalistas, eurocentristas, propios del MCMC en base al cual han sido creadas. La exclusión de todo aquello que no se adapta a sus parámetros, es característica intrínseca del MCMC, encarnado fuertemente en las universidades. En Argentina, estas características de la modernidad coexisten en las universidades con políticas como el acceso irrestricto y la gratuidad, que crean la ilusión de que la universidad es accesible para todos y todas.

Sin embargo, el principio de igualdad de oportunidades termina por generar una inclusión excluyente, en tanto no contempla las desigualdades de base $y$, por ende, las reproduce. Proponemos, entonces, desde la opción decolonial, la posibilidad de pensar en una inclusión una en términos interculturales, que se aleje de la lógica unidireccional y favorezca el diálogo intercultural, permitiendo incluirnos en múltiples sentidos.

La inclusión excluyente presente en las universidades argentinas, a la que hemos complejizado como una inclusión colonial, genera que muchos estudiantes provenientes de grupos en situación de vulnerabilidad no puedan permanecer en las universidades. La respuesta del Estado y las propias universidades a esta situación se centra en la promoción de políticas focalizadas, principalmente materializadas en becas y en sistemas de tutorías. Estas estrategias son las que denominamos de retención, mientras que resaltamos la importancia de focalizar en las experiencias estudiantiles, es decir, en las estrategias que llevan adelante los propios estudiantes 
Universidad y universalidad: aportes de la opción decolonial al estudio de políticas universitarias para la inclusión de estudiantes indígenas

para permanecer en las universidades. Así, destacamos la importancia de las investigaciones situadas.

De este panorama general, para nuestra investigación interesa centralmente las experiencias de los estudiantes que se autorreconocen como indígenas. Al respecto, problematizamos la ausencia de políticas estatales para atender a las demandas de estos jóvenes respecto al derecho a la educación superior. Sin embargo, mencionamos que la autonomía que poseen las universidades argentinas permite que en algunas de ellas se hayan aprobado proyectos destinados a los estudiantes indígenas, llamados IBE. Éstas son experiencias que, tomando distintas características, permiten avizorar nuevos horizontes, críticos al MCMC. Para avanzar hacia ellos, consideramos que es necesario analizar las IBE desde las experiencias de los propios estudiantes indígenas. A tal fin, la opción decolonial sirve como un enfoque epistemológico pertinente.

\section{Bibliografía}

Artieda, T.; Rosso, L.; Luján, A. y Zamora, A. L. (2017). Programa Pueblos Indígenas de la Universidad Nacional del Nordeste. Algunas reflexiones y propuestas en torno a la inclusión y la interculturalidad. En D. Mato, (comp.) Educación Superior y Pueblos Indígenas y Afrodescendientes en América Latina. Políticas y prácticas de democratización, interculturalización e inclusión. Buenos Aires: EDUNTREF.

Buchbinder, P. (2006) La Universidad: breve introducción a su evolución histórica. Universidad Nacional del Litoral. Disponible en: http://www.unlvirtual.edu.ar/wpcontent/recursos/La universidad breve evolucion historica.pdf.

Capelari, M. I. (2016) El rol del tutor en la Universidad: configuraciones, significados y prácticas. Buenos Aires: SB.

Carli, S. (2012). El estudiante universitario: Hacia una historia del presente de la educación pública. Buenos Aires: Siglo XXI.

Carli, S. (2014). Universidad pública y experiencia estudiantil. Historia, política y vida cotidiana. Buenos Aires: Editorial Miño y Dávila. 
Civallero, E. (2007). Bibliotecas en comunidades indígenas: Guía de acción y reflexión. Córdoba: Wayrachaki.

Cloud, L.; González, V. y Lacroix, L. (2019) Categorías, denominaciones y derechos vinculados al indigenismo en América Latina. Variaciones históricas y desafíos actuales. En Bellier, Irene (Comp.) Pueblos Indígenas en el Mundo. Buenos Aires: EUDEBA

Coronado, M., y Gómez Boulin, M. J. (2015). Orientación, tutorías y acompañamiento. En Educación Superior. Análisis de las trayectorias estudiantiles. Los jóvenes ante sus encrucijadas. Buenos Aires: Noveduc.

Cupo, Vicente (2010) Orígenes históricos de la universidad. En Toribio, D. (Comp.) LA universidad en la Argentina. Miradas sobre su evolución y perspectivas. Remedios de Escalada: Ediciones de la UNLa.

Czarny, G., Ossola, M. y Paladino, M. (2018). Presentación al dossier Jóvenes indígenas y universidades en América Latina: sentidos de la escolarricas, diversidad de experiencias y retos de la profesionalización. Antropología Andina Muhunik Jathasa $5(1)$.

Diez, Cecilia; García, Javier; Montesinos, María Paula; Pallma, Sara; Paoletta, Hotacio. (2015). Discutiendo categorías... acerca de los usos (y abusos) de los términos inclusión y exclusión. Boletín de Antropología y Educación (9), 33-39.

Diez, M. L. (2004). Reflexiones en torno a la interculturalidad. Cuadernos de Antropología social(19), 191-213.

Dussel, E. (2000). Europa, modernidad y eurocentrismo. En La colonialidad del saber: eurocentrismo y ciencias sociales. Perspectivas (p. 24-33). Buenos Aires: CLACSO, Consejo Latinoamericano de Ciencias Sociales.

Ezcurra, A. M. (2011). Masificación y enseñanza superior: una inclusión excluyente. En N. Fernández Lamarra y M. Costa de Paula (comps.) La democratización de la educación superior en América Latina. Buenos

Freire, P. (1987). Pedagogía del oprimido (36a. ed.). México: Siglo XXI. 
Universidad y universalidad: aportes de la opción decolonial al estudio de políticas universitarias para la inclusión de estudiantes indígenas

García De Fanelli, A. (2014). Inclusión Social en la Educación Superior argentina: Indicadores y políticas en torno al acceso y a la graduación. Pág. Educ. 7(2), 124-151.

García De Fanelli, A. (2015) La cuestión de la graduación en las universidades nacionales de la Argentina: Indicadores y políticas públicas a comienzos del siglo XX. Propuesta Educativa, 43, 17-31

González, F. (2020) Producción y circulación de sentidos en la configuración de las dimensiones supra e infra hábitat. Experiencias de producción de hábitat en la Puna y en el Chaco salteños a partir de proyectos de extensión con la comunidad Kolla de Hurcuro y el pueblo Wichí de El Cocal (Salta, 2017-2018). Tesis Doctoral. Córdoba: Universidad Nacional de Córdoba.

Guaymás, A. (2016). Educación superior y pueblos indígenas: políticas y prácticas de reconocimiento de la diversidad cultural e interculturalidad en Argentina. En M. V.; Di Caudo, D. Llanos Erazo y M. Ospina, (coords.): Interculturalidad y Educación desde el Sur. Contextos, experiencias y voces. Quito: Editorial Universitaria Abya Yala.

Hanne, V. (2015) “Diversidad cultural, Universidad e Inclusión socio-educativa. Una aproximación a dimensiones institucionales y psicosociales de influencia en las trayectorias académicas de estudiantes de pueblos indígenas (UNCuyo UNSa)". Tesis Doctoral. Mendoza: Universidad Nacional de Cuyo

Hanne, V. (2018) Estudiantes indígenas y Universidad: realidades y retos ante la diversidad cultural. Caso de la Universidad Nacional de Salta. Alteridad, 13(1), 14-29. https://doi.org/10.17163/alt.v13n1.2018.01.

Jonsson, S. (2010). La ideología del universalismo. NRL (63), 111-122.

Lander (2000) La colonialidad del saber: eurocentrismo y ciencias sociales. Perspectivas Latinoamericanas. Buenos Aires: CLACSO

Luciano, G. (2009). Indígenas no Ensino Superior: Novo Desafio para as Organizações Indígenas e Indigenistas no Brasil. En Congreso Internacional de Americanistas. México, D.F. 
Luján, A.; Soto, M. y Rosso, L (2018). Experiencias de ingreso y permanencia de estudiantes indígenas en una universidad convencional del nordeste argentino. Antropología Andina Muhunchik - Jathasa Vol. 4 Núm. 1.

Mato, D. (coord.). (2015). Educación Superior y Pueblos Indígenas en América Latina. Buenos Aires: EDUNTREF.

Mignolo, W. (2007) El pensamiento decolonial: desprendimiento y apertura. En CastroGómez, S. y Grosfoguel, R. (comp.) El giro decolonial: reflexiones para una diversidad epistémica más allá del capitalismo global. Bogotá: Siglo del Hombre Editores, Universidad Central, Instituto de Estudios Sociales Contemporáneos y Pontificia Universidad Javeriana, Instituto Pensar.

Ossola, M. M. (2013). Jóvenes wichí en la educación superior de Salta: interpelaciones escolares, étnicas y etarias. (Tesis Doctoral). Buenos Aires: UBA.

Palermo, Z. (2014) Irrupción de saberes "otros" en el espacio pedagógico: hacia una "democracia decolonial". En Borsani, M. E. y Quintero, P. (Comp.) Los desafíos decoloniales de nuestros días: pensar en colectivo. Neuquén: EDUCO Universidad Nacional del Comahue.

Quijano, A. (1992). Colonialidad y modernidad-racionalidad. En H. Bonilla (ed.) Los conquistados. 1942 y la población indígena de las Américas. Bogotá: Tercer Mundo.

Quijano, A. (2000). Colonialidad del poder, eurocentrismo y América Latina. En E. Lander (comp.), La colonialidad del saber, eurocentrismo y Ciencias sociales. Buenos Aires: CLACSO.

Reygadas, L. (2007). La desigualdad después del (multi)culturalismo. En A. Giglia, C. Garma y A. P. de Teresa (comps.) ¿A dónde va la antropología? México: Universidad Autónoma Metropolitana Iztapalapa.

Rodríguez y Sulca (2020) El acceso a la educación y la inclusión socioeducativa de estudiantes indígenas. Provincia de Salta-Argentina. TEXTURA - Revista de Educação e Letras, v. 22, n. 51, 194-218. https://doi.org/10.17648/textura2358-0801-v22n51-5683 
Universidad y universalidad: aportes de la opción decolonial al estudio de políticas universitarias para la inclusión de estudiantes indígenas

Rodríguez, N. (2018) Problematizaciones en torno al concepto de universalidad: del eurocentrismo a la interculturalidad; Universidad Nacional de Salta. Facultad de Humanidades; Cuadernos de Humanidades; 29; 12-2018; 201-210

Rodríguez, N. y Ossola, M. M. (2019). Tutoría universitaria y educación intercultural:

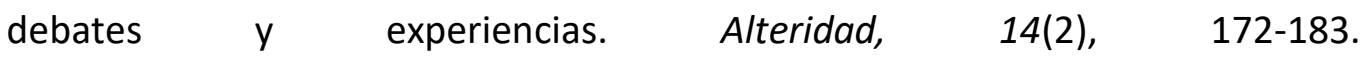
https://doi.org/10.17163/alt.v14n2.2019.02

Rosso (2019). Universidades convencionales y participación indígena. Análisis de una experiencia en Chaco, Argentina. Tellus 38. doi: https://doi.org/10.20435/tellus.v19i38.593.

Rosso, L.; Artieda, T y Luján, A (2016). Universidad y Pueblos Indígenas del Chaco. Análisis de una política de inclusión, participación e interculturalidad. En D. Mato, (coord.) Educación Superior y Pueblos Indígenas en América Latina. Experiencias, interpelaciones y desafíos. Buenos Aires: EDUNTREF.

Santana Colin, Y. (2018). Formación académica y militancia de los intelectuales indígenas mexicanos. Antropología Andina Muhunchik - Jathasa. 4(1).

Soto, M. G.; Luján, A. E. y Rosso, L. L. (2019) Nuevas lecturas a la permanencia de estudiantes indígenas en una Universidad Convencional argentina. Ponencia presentada en el 3er Congreso Internacional de Pueblos Indígenas de América Latina. Brasilia, 3-5 julio.

Stavenhagen, R. (2007). Los pueblos indígenas y sus derechos. México: UNICEF.

Terigi, F. (2008) En la perspectiva de las trayectorias escolares. Comentario al capítulo 3 del atlas de las desigualdades educativas en américa latina: "la asistencia escolar en la actualidad. trayectorias educativas en 8 países de américa latina". Buenos Aires: SITEAL - IIPE - UNESCO Sede Regional Buenos Aires.

Tintaya Condorí, P. (2003). Utopías e Interculturalidad: Motivación en niños aymaras. La Paz: Instituto de Estudios Bolivianos.

Trinchero, H. H. (2010). Los pueblos originarios en Argentina. Representaciones para una caracterización problemática. Cultura y representaciones sociales, 4(8), 111-139. 
Walsh, C. (2010). Interculturalidad Crítica y Educación Intercultural. En J. Viña, L. Tapia y C. Walsh (comps.) Construyendo Interculturalidad Crítica. La Paz: Instituto Internacional de Integración del Convenio Andrés Bello.

Walsh, C. (2014). Interculturalidad y Colonialidad del Poder. Un pensamiento y posicionamiento otro desde la diferencia colonial. En W. Mignolo (comp.) Interculturalidad, decolonización del estado y del conocimiento. Buenos Aires: Del signo.

Wigdorovitz de Camilloni, A. R. (2008). El concepto de inclusión educativa: definición y redefiniciones. Políticas Educativas-Campinas, 2(1), 1-12. (goo.gl/bUJrWw) (2019-01-28) 